ARTICLE

https://doi.org/10.1038/s41467-019-08610-6

\title{
Suppressed phase separation of mixed-halide perovskites confined in endotaxial matrices
}

Xi Wang (1) ${ }^{1}$, Yichuan Ling 1 , Xiujun Lian', Yan Xin², Kamal B. Dhungana ${ }^{3}$, Fernando Perez-Orive ${ }^{1}$, Javon Knox ${ }^{1}$ Zhizhong Chen ${ }^{4}$, Yan Zhou ${ }^{5}$, Drake Beery ${ }^{5}$, Kenneth Hanson ${ }^{5,6}$, Jian Shi ${ }^{4}{ }^{4}$, Shangchao Lin ${ }^{3,6,7}$ \& Hanwei Gao $1,2,6$

The functionality and performance of a semiconductor is determined by its bandgap. Alloying, as for instance in $\ln _{x} \mathrm{Ga}_{1-x} \mathrm{~N}$, has been a mainstream strategy for tuning the bandgap. Keeping the semiconductor alloys in the miscibility gap (being homogeneous), however, is non-trivial. This challenge is now being extended to halide perovskites - an emerging class of photovoltaic materials. While the bandgap can be conveniently tuned by mixing different halogen ions, as in $\operatorname{CsPb}\left(\left.\mathrm{Br}_{x}\right|_{1-x}\right)_{3}$, the so-called mixed-halide perovskites suffer from severe phase separation under illumination. Here, we discover that such phase separation can be highly suppressed by embedding nanocrystals of mixed-halide perovskites in an endotaxial matrix. The tuned bandgap remains remarkably stable under extremely intensive illumination. The agreement between the experiments and a nucleation model suggests that the size of the nanocrystals and the host-guest interfaces are critical for the photo-stability. The stabilized bandgap will be essential for the development of perovskite-based optoelectronics, such as tandem solar cells and full-color LEDs.

\footnotetext{
${ }^{1}$ Department of Physics, Florida State University, Tallahassee, FL 32306, USA. ${ }^{2}$ Condensed Matter Science, National High Magnetic Field Laboratory, Tallahassee, FL 32310, USA. ${ }^{3}$ Department of Mechanical Engineering, Florida State University, Tallahassee, FL 32310, USA. ${ }^{4}$ Department of Materials Science and Engineering, Rensselaer Polytechnic Institute, Troy, NY 12180, USA. ${ }^{5}$ Department of Chemistry and Biochemistry, Florida State University, Tallahassee, FL 32306, USA. ${ }^{6}$ Materials Science \& Engineering Program, Florida State University, Tallahassee, FL 32306, USA. ${ }^{7}$ School of Mechanical and Power Engineering, Shanghai Jiao Tong University, 200240 Shanghai, China. Correspondence and requests for materials should be addressed to H.G. (email: hgao3@fsu.edu)
} 
T he earth-abundant composition and the simple synthetic procedures make halide perovskites promising for the effective optoelectronics ${ }^{1-8}$, such as solution-processed high-efficiency solar cells ${ }^{9-13}$ and high-brightness light emitting diodes ${ }^{14-18}$. Besides other desirable optical and electrical properties, the halide perovskites offer a unique opportunity to optimize the spectral range of photoresponse by tuning the bandgaps ${ }^{19,20}$. Two types of halogen anions can be accommodated in homogenous crystal lattices with nearly arbitrary mixing ratios, leading to a wide range of the bandgap tunability. For example, the bandgap of the perovskite $\mathrm{CsPb}\left(\mathrm{Br}_{x} \mathrm{I}_{1-\mathrm{x}}\right)_{3}$ can be tuned continuously from $2.4 \mathrm{eV}$ (corresponding to $\mathrm{CsPbBr}_{3}$ ) to $1.75 \mathrm{eV}$ (corresponding to $\mathrm{CsPbI}_{3}$ ), covering half of the visible spectrum. The range could be further extended when chlorine is incorporated ${ }^{19,20}$. In optoelectronic applications, the bandgap of photoactive semiconductors is often critical to device performance-it determines the upper limit of the energy conversion efficiency in solar cells ${ }^{21}$, or the color of emission in light emitting diodes $^{17,18}$.

The practical value of the bandgap tunability in perovskites, however, has been limited due to poor material stability under optical illumination ${ }^{22-28}$. While the freshly prepared mixedhalide perovskites exhibit homogenous crystal phases, the bandgap could vary significantly under continuous optical illumination. Various measurements indicate that the observed bandgap shifts could be attributed to photoinduced phase separation $22-28$. The homogeneously distributed halogen anions segregated into bromine- and iodine-rich domains, exhibiting a redshifted photoluminescence peak and split X-ray diffraction patterns ${ }^{22}$. A few studies show that such photoinduced degradation could be mitigated in some of the mixed-halide perovskites with certain compositions ${ }^{18,29}$. Yet, stabilizing the bandgap across the entire spectrum (from pure bromide to pure iodide, i.e. $0<x<1$ ) remains challenging. In this work, we find that the light-induced phase separation could be effectively suppressed when crystals of the mixed-halide perovskite $\mathrm{CsPb}\left(\mathrm{Br}_{\mathrm{x}} \mathrm{I}_{1-\mathrm{x}}\right)_{3}$ are spatially confined in an endotaxial matrix $\mathrm{Cs}_{4} \mathrm{~Pb}\left(\mathrm{Br}_{\mathrm{x}} \mathrm{I}_{1-\mathrm{x}}\right)_{6}$. Stabilization of the tuned bandgap is achieved across the full spectrum (regardless of the $\mathrm{Br}$ / I ratio). It is remarkable that, for certain composition $(x \leq 0.6)$, the tuned bandgaps remain stable under extremely intensive illumination up to $440 \mathrm{~W} \mathrm{~cm}^{-2}$ (as a reference, the standard solar spectrum (AM1.5) has an integrated power density of $0.1 \mathrm{~W} \mathrm{~cm}$ ${ }^{-2}$ ). Note that such intensity is about an order of magnitude higher than what is typically used in concentrator solar cells ${ }^{30}$. The observed phenomena are consistent with the predictions from a thermodynamic model based on nucleation in solids. In both experiments and theory, the size of the crystallites and the host-guest cohesive energy, are found to be critical factors to the photo-stability of the mixed-halide perovskites.

\section{Results}

Correlation between the phase stability and the morphology. We prepared thin films of mixed-halide perovskites using dualsource thermal evaporation (Methods). Solid precursors $\mathrm{PbBr}_{2} /$ $\mathrm{PbI}_{2}$ and $\mathrm{CsBr} / \mathrm{CsI}$ were evaporated onto glass substrates followed by thermal annealing for sufficient inter-diffusion and reactions. With a stoichiometry ratio of $\mathrm{Pb} / \mathrm{Cs}$ in the premixed precursors, polycrystalline thin films of $\mathrm{CsPb}\left(\mathrm{Br}_{\mathrm{x}} \mathrm{I}_{1-\mathrm{x}}\right)_{3}$ was obtained (Fig. 1a). The perovskite crystal phase was confirmed consistently in the selected area diffraction (SAD) patterns (Fig. 1a, inset), the highresolution transmission electron microscopy (TEM) images, and the corresponding fast Fourier transformed (FFT) patterns (Fig. 1b). The photoluminescence peak (corresponding to the energy of bandgap) of $\mathrm{CsPb}\left(\mathrm{Br}_{\mathrm{x}} \mathrm{I}_{1-\mathrm{x}}\right)_{3}$ could be tuned by varying the ratio of $\mathrm{Br} / \mathrm{I}$ in the premixed precursors (Fig. 1c).
Photoluminescence of all these $\operatorname{CsPb}\left(\mathrm{Br}_{\mathrm{x}} \mathrm{I}_{1-\mathrm{x}}\right)_{3}$, however, appeared unstable under extended photoexcitation (Fig. 1c). The peak position, regardless of its initial wavelength, redshifted to $\sim 1.87 \mathrm{eV}$ within $10 \mathrm{~min}$ of illumination at an intensity of 0.3 $\mathrm{W} \mathrm{cm}-2$ (compared with an integrated power density of $0.1 \mathrm{~W}$ $\mathrm{cm}^{-2}$ of the AM1.5 standard solar spectrum). Such redshift, corresponding to a reduction in the bandgap, is consistent with previously reported observations ${ }^{23}$. The initially homogeneous $\mathrm{CsPb}\left(\mathrm{Br}_{\mathrm{x}} \mathrm{I}_{1-\mathrm{x}}\right)_{3}$ segregated into $\mathrm{Br}$ - and I-rich domains with increased and decreased bandgaps, respectively. The energy transfer between neighboring domains makes the smallbandgap species (i.e. the I-rich domains) dominate the photoluminescence after the phase separation ${ }^{22-24}$. The exceptions in our experiments are the samples with very large $\mathrm{Br}$ content (Fig. 1c, $X_{\mathrm{Br}}=0.8$ and 0.9 ), which exhibited photoluminescence from both large- and small-bandgap species $(2.4 \mathrm{eV}$ and $1.87 \mathrm{eV}$, respectively). The amount of the $\mathrm{Br}$-rich domains in this case is likely too large to be quenched completely by the I-rich domains.

The photoinduced phase separation is found to be highly suppressed when the mixed-halide perovskites are created in form of nanocrystals and are embedded in a non-perovskite matrix. We obtained such host-guest structures by simply increasing the relative weight ratio of the Cs-containing precursor (i.e. $\mathrm{CsBr} / \mathrm{CsI}$ ). According to the phase diagram ${ }^{31}$, such variation of the precursor ratio would produce a mixture of $\mathrm{CsPb}\left(\mathrm{Br}_{\mathrm{x}} \mathrm{I}_{1-\mathrm{x}}\right)_{3}$ and $\mathrm{Cs}_{4} \mathrm{~Pb}\left(\mathrm{Br}_{\mathrm{x}} \mathrm{I}_{1-\mathrm{x}}\right)_{6}$ when fully reacted. The expected composite structure was confirmed using TEM (Fig. 1d). The electron diffraction pattern was predominately from the hexagonal lattices of the $\mathrm{Cs}_{4} \mathrm{~Pb}\left(\mathrm{Br}_{\mathrm{x}} \mathrm{I}_{1-\mathrm{x}}\right)_{6}$ single crystal (close to [100]). A faint ring corresponding to the $\{200\}$ cubic plane of $\mathrm{CsPb}\left(\mathrm{Br}_{\mathrm{x}} \mathrm{I}_{1-\mathrm{x}}\right)_{3}$ indicated that many tiny crystallites of the perovskite were distributed within the 400-nm electron beam spot (Fig. 1d, inset). The high-resolution TEM image showed that the nanocrystal $\mathrm{CsPb}\left(\mathrm{Br}_{\mathrm{x}} \mathrm{I}_{1-\mathrm{x}}\right)_{3}$, with an average radius of $\sim 7.5 \mathrm{~nm}$, were embedded in the $\mathrm{Cs}_{4} \mathrm{~Pb}\left(\mathrm{Br}_{\mathrm{x}} \mathrm{I}_{1-\mathrm{x}}\right)_{6}$ matrix with clear Moiré Fringes (Fig. 1e). The photoluminescence from these isolated, spatiallyconfined perovskites appeared remarkably stable. After continuous illumination for 5 hours $\left(0.3 \mathrm{~W} \mathrm{~cm}{ }^{-2}, 365 \mathrm{~nm}\right.$ wavelength), the emission wavelength remained unchanged in all the samples regardless of the Br/I mixing ratio (Fig. 1f). Such photo-stability was only reported previously in iodine dominated mixed-halide perovskites $(x<0.3)$ in rare cases $^{23}$.

A thermodynamic model based on nucleation theory ${ }^{32}$ was developed to understand the mechanisms of the high photostability observed (Supplementary Note 1, Theoretical Model Based on Nucleation). In this model, whether the mixed-halide perovskites would remain homogeneous or experience phase separation is determined by changes of the Gibbs free energy $\Delta G$. The homogenous phase would be stable when $\Delta G$ is negative.

$\Delta G$ in the dark $\left(\Delta G_{\text {dark }}\right)$ is contributed together by the volumetric enthalpy $\left(\Delta h_{\text {mix }}\right)$, the volumetric entropy term $\left(T \Delta s_{\text {mix }}\right)$ and the cohesive energies $\Sigma c_{i} W_{i} r^{2}$ :

$$
\Delta G_{\text {dark }}\left(X_{\mathrm{Br}}, T\right)=\frac{4}{3} \pi r^{3}\left[\Delta h_{\text {mix }}\left(X_{\mathrm{Br}}, T\right)-T \cdot \Delta s_{\text {mix }}\left(X_{\mathrm{Br}}, T\right)\right]-\sum_{i} c_{i} W_{i} r^{2}
$$

The terms are functions of the average grain size $r$, the temperature $T$, and the relative amount of bromine in the mixed-halide phase $X_{\mathrm{Br}}$. Based on the quantities calculated using Density Functional Theory (Supplementary Note 2, Computational Methods), $\Delta G_{\text {dark }}$ is found to be negative regardless of the mixed-halide composition (Fig. 2a). A relatively large domain size $r=35 \mathrm{~nm}$ was assumed in the calculation, but the results would not change qualitatively even if a larger $r$ was used. The theoretical predictions are consistent with the experimental results observed in this work and reported previously: the photoluminescence spectra (Fig. 1c), the X-ray diffraction 

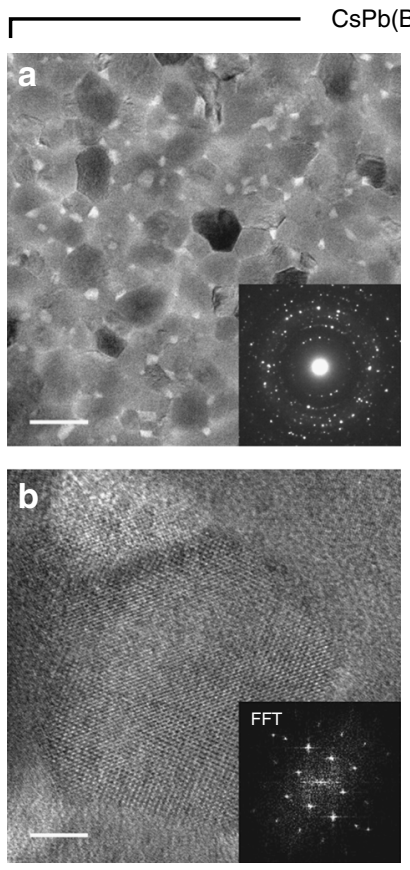

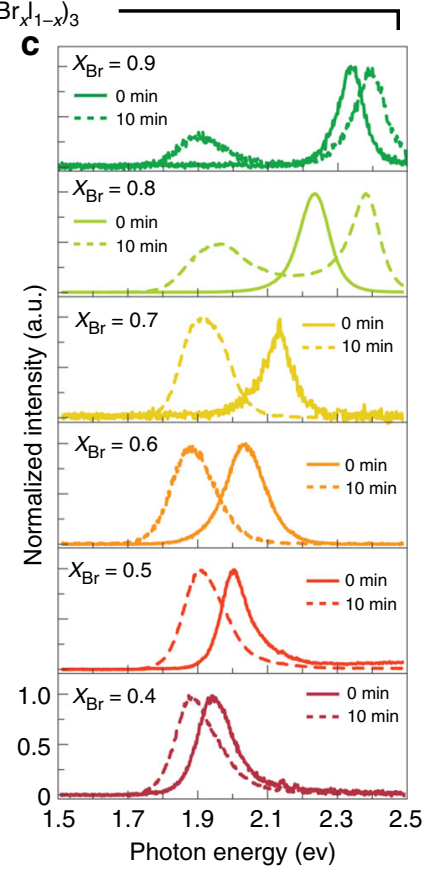

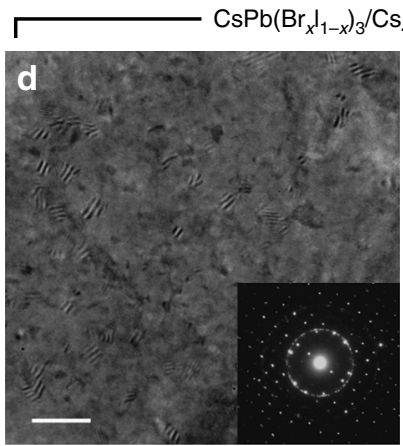

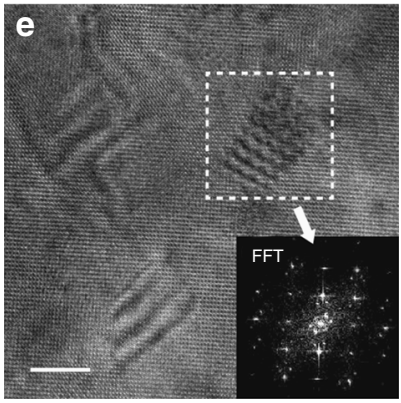

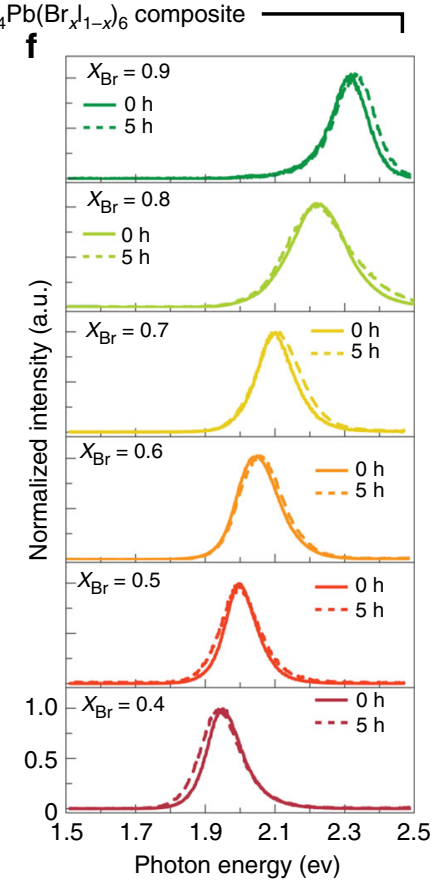

Fig. 1 The phase stability of mixed-halide perovskite $\operatorname{CsPb}\left(\left.\mathrm{Br}_{x}\right|_{1-x}\right)_{3}$ is correlated with the morphology. a A representative TEM image of polycrystalline $\mathrm{CsPb}\left(\mathrm{Br}_{x} \mathrm{I}_{1-\mathrm{x}}\right)_{3}$ thin films showed an average domain size around $r=35 \mathrm{~nm}$. $\mathbf{b}$ An HR-TEM image of a single domain and the corresponding FFT pattern of the image (inset) confirmed these thin films were composed of perovskites. $\mathbf{c}$ The photoluminescence peak of these mixed-halide perovskites shifted exclusively to $1.87 \mathrm{eV}$ after continuous illumination. The solid lines were the spectra taken from freshly made samples and the dashed lines were measured after 10 min illumination with the intensity of $0.3 \mathrm{~W} \mathrm{~cm}^{-2}$. $\mathbf{d}$ In composite thin films, $\mathrm{CsPb}\left(\mathrm{Br}_{x} \mathrm{I}_{1-x}\right)_{3}$ and $\mathrm{Cs}_{4} \mathrm{~Pb}\left(\left.\mathrm{Br}_{x}\right|_{1-x}\right)_{6}$ formed host-guest structures. Inset:

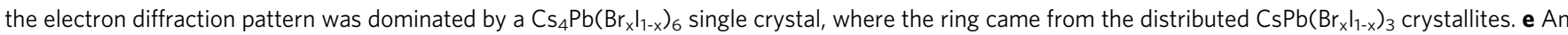
HR-TEM image with clear Moiré Fringes showed an average $\operatorname{CsPb}\left(\mathrm{Br}_{x} \mathrm{I}_{1-x}\right)_{3}$ domain size about $r=7.5 \mathrm{~nm}$. Inset: the FFT pattern of the highlight area

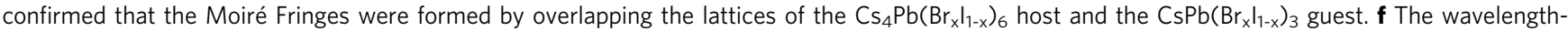
tunable photoluminescence from the $\mathrm{CsPb}\left(\left.\mathrm{Br}_{x}\right|_{1-x}\right)_{3}$ crystallites in the composites exhibited high stability under $0.3 \mathrm{~W} \mathrm{~cm}{ }^{-2}$ illumination. The scale bars in $\mathbf{a}$ and $\mathbf{d}$ are $100 \mathrm{~nm}$ and $50 \mathrm{~nm}$, respectively. The scale bars in $\mathbf{b}$ and $\mathbf{e}$ are $10 \mathrm{~nm}$. Magnified figures of the insets can be found in Supplementary Figure 8
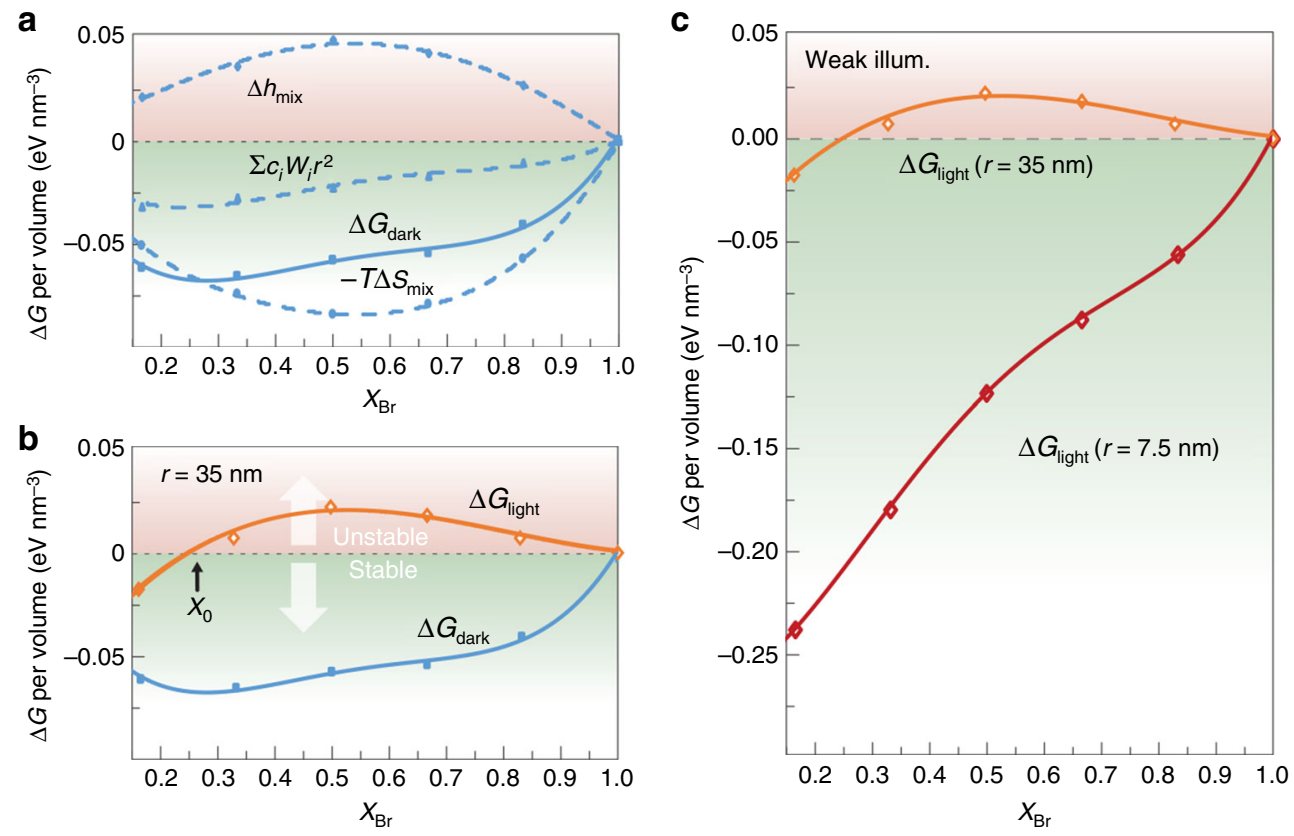

Fig. $2 \mathrm{~A}$ thermodynamic nucleation model explains the dependence of phase stability on the morphology. a The calculated $\Delta G_{\text {dark }}$ per volume (solid line) is negative regardless of the $\mathrm{Br}$ content. The dashed lines show the calculated volumetric enthalpy $\Delta h_{\text {mix }}$ volumetric entropy term $T \Delta s_{\text {mix }}$ and cohesive energy $\Sigma c_{i} W_{i} r^{2}$. A relatively large grain size was used here $(r=35 \mathrm{~nm})$. b Under illumination, the calculated free energy $\Delta \mathrm{G}_{\text {light }}$ becomes partially positive assuming the same grain size. A threshold composition $X_{0} \sim 0.3$ divides the mixed-halide perovskites into stable (I-rich) and unstable (Br-rich) regions. $\mathbf{c}$ To mimic the experimental conditions of the $\mathrm{CsPb}\left(\left.\mathrm{Br}_{x}\right|_{1-x}\right)_{3} / \mathrm{Cs}_{4} \mathrm{~Pb}\left(\left.\mathrm{Br}_{x}\right|_{1-x}\right)_{6}$ composites, a small grain size $(r=7.5 \mathrm{~nm})$ was assumed and the cohesive energy was considered. The calculated $\Delta G_{\text {light }}$ turns negative, indicating a stable phase of homogenous mixed-halide perovskites 
patterns, and the UV-VIS absorption spectra (Supplementary Figure 1) all indicated that the mixed-halide perovskites remained homogeneous in the dark.

Optical illumination could turn the $\Delta G$ to be favorable for phase separation under certain conditions. The effects of photoexcitation in the model were accounted for by involving the photoinduced polarons ${ }^{24,33-35}$. Polarons could bring in excessive strain energy $\left(\Delta g_{s}\right)$ due to the locally shortened and lengthened $\mathrm{Pb}$-halogen bonds (Supplementary Figure 2):

$$
\Delta G_{\text {light }}\left(X_{\mathrm{Br}}, T\right)=\Delta G_{\text {dark }}\left(X_{\mathrm{Br}}, T\right)+\frac{4}{3} \pi r^{3} \cdot \Delta g_{\mathrm{s}}\left(X_{\mathrm{Br}}\right)
$$

When $\Delta g_{\mathrm{s}}$ becomes sufficiently large, the strain energy would be released by de-mixing the halogen anions and forming $\mathrm{Br}$ - and Irich domains (i.e. phase separation).

$\Delta G_{\text {light }}$, assuming weak illumination, is found positive when $X_{\mathrm{Br}}>0.3$ (Fig. 2b). The negative $\Delta G_{\text {light }}$ in the I-rich region is indicative of relatively better stability and stemmed from the larger interfacial area with nucleated I-rich domain with a fixed crystal size. The calculated threshold $X_{0}$ between the stable and unstable compositions is in reasonable agreement with those observed in our measurements (Fig. 1c) and reported by others previously ${ }^{23}$ : photoluminescence from pure $\mathrm{Cs} \mathrm{Pb}\left(\mathrm{Br}_{\mathrm{x}} \mathrm{I}_{1-\mathrm{x}}\right)_{3}$ redshifted notably under continuous optical illumination when $X_{\mathrm{Br}}>$ 0.3 . The coincidence testified the reasonableness of the physical quantities that we obtained using the nucleation model. It is worth noting that the photoinduced phase separation is reversible in experiments. The shifted photoluminescence would gradually return to its initial wavelength after the optical illumination was removed. The reversibility is also a natural prediction in our theory given that the model was built entirely on the thermodynamic basis.

The phase separation under optical illumination could alternatively be suppressed if the domain size of the mixedhalide perovskites is reduced (Supplementary Note 1.3). The stability could be attributed to the dominating role of the cohesive energy $\left(\Sigma c_{i} W_{i} r^{2}\right)$ when the surface-to-volume ratio is increased. While all other terms in the model are proportional to the volume of the domains $\left(\propto r^{3}\right)$, the cohesive energy is a function of the area $\left(\propto r^{2}\right)$ (Equation (1) \& (2) in main text and Supplementary Equation $1 \sim 3$ ). Domains of the new phase (i.e. the iodine-rich perovskite domains) nucleated out of the mixed-halide perovskites would need to reach a certain critical size $r^{*}$ in order for the volume-proportional terms to dominate and the phase separation to prevail $\left(-\Delta G_{\text {light }}<0\right)$. In other words, if the mixed-halide perovskites are confined with an average domain size smaller than $r^{*}$, nucleation and growth of iodine-rich perovskite domains would never be energy favorable. In that case, the infinitesimal domains of the new I-rich phase would experience dynamic equilibrium between nucleation and dissolution without prevailing phase separation. Experimentally, by confining the nanocrystals of mixed-halide perovskites with $r=7.5 \mathrm{~nm}$ in the nonperovskite matrix, we were able to limit the size of iodine-rich nucleates. The phase separation then would not occur regardless of the $\mathrm{Br}$ content $X_{\mathrm{Br}}$ (Fig. 2c). The predicted phase stability agrees well with our experiments, in which nanocrystals of the perovskite $\mathrm{CsPb}\left(\mathrm{Br}_{\mathrm{x}} \mathrm{I}_{1-\mathrm{x}}\right)_{3}$ were spatially confined in the $\mathrm{Cs}_{4} \mathrm{~Pb}$ $\left(\mathrm{Br}_{\mathrm{x}} \mathrm{I}_{1-\mathrm{x}}\right)_{6}$ matrix with large surface-to-volume ratio (Fig. 1d-f). What also helps to suppress the phase separation is the endotaxial lattice matching between the $\mathrm{CsPb}\left(\mathrm{Br}_{\mathrm{x}} \mathrm{I}_{1-\mathrm{x}}\right)_{3}$ guest and the $\mathrm{Cs}_{4} \mathrm{~Pb}$ $\left(\mathrm{Br}_{\mathrm{x}} \mathrm{I}_{1-\mathrm{x}}\right)_{6}$ host matrix ${ }^{31}$. The phase separation becomes further unfavorable because a segregated perovskite domain would increase the energy at the host-guest interfaces and raise the total Gibbs free energy (Supplementary Figure 3b).

Spectral stability under extremely intensive illumination. Remarkably, the tuned bandgap of the $\mathrm{CsPb}\left(\mathrm{Br}_{\mathrm{x}} \mathrm{I}_{1-\mathrm{x}}\right)_{3} / \mathrm{Cs}_{4} \mathrm{~Pb}$ $\left(\mathrm{Br}_{\mathrm{x}} \mathrm{I}_{1-\mathrm{x}}\right)_{6}$ composites remained stable even under extremely intensive illumination. Using a focused laser beam $(\lambda=405 \mathrm{~nm})$, we strained the samples optically with an intensity $440 \mathrm{~W} \mathrm{~cm}^{-2}$ (equivalent to 4400 times of integrated intensity of the AM1.5 solar irradiation). No obvious changes were observed in the photoluminescence spectra taken from the samples with $X_{\mathrm{Br}} \leq 0.6$ (Fig. 3a-c). However, with higher bromine concentrations, the samples started to show phase separation under the strong illumination condition (Fig. 3d-f). The correlation between the photo-stability and the composition is consistent with the relative phase stability between the Br- and I-rich perovskites discussed above (Fig. 2c) and in Supplementary Figure 4. $\Delta G_{\text {light }}$ becomes smaller with less $\mathrm{Br}$ content $\left(X_{\mathrm{Br}}\right)$ and results in a more stable alloy phase. Note that the amount of peak shift appeared noticeably different between the composites and the pure perovskites: the peak in the former settled at higher energy $(2.00 \mathrm{eV})$ compared with the latter (Fig 1c, $1.87 \mathrm{eV})$. The quantitative difference suggested a smaller degree of phase separation in the composites with a smaller change of iodine concentration in the segregated I-rich domains.

The observed high photo-stability indicates that the cohesive energy is a more dominating factor in stabilizing a homogenous phase. As long as the grain size of the mixed-halide perovskites is sufficiently small, the presumable increase of the cohesive energy associated with the phase separation would dominate over the increase of the photoinduced strain energy, leading to a highly stable homogenous phase even though the high illumination intensity was assumed (Supplementary Note 1.4).

Temperature dependent photo-stability. Photo-stability of the mixed-halide perovskites, according to the theoretical model with

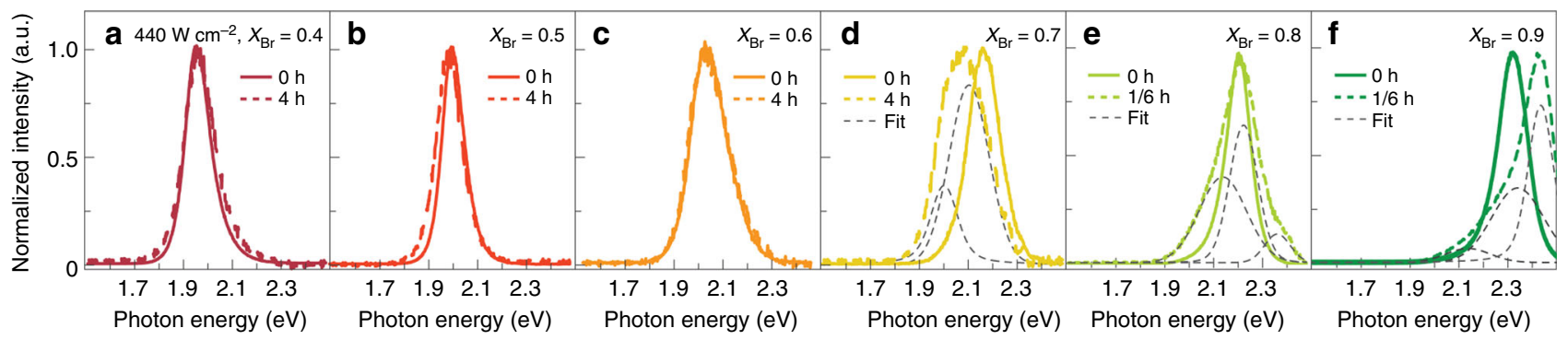

Fig. 3 The perovskite composites remained stable under extremely intensive illumination. The solid and dashed lines are spectra obtained from experiments. The thin grey lines show the results of multiple-peak fitting. a-c Minimal changes were observed in the photoluminescence measured from the samples with $X_{\mathrm{Br}} \leq 0.6$, even after 4 hours of illumination with intensity of $440 \mathrm{~W} \mathrm{~cm}^{-2}$. $\mathbf{d}-\mathbf{f}$ The sample with higher bromine content $\left(X_{\mathrm{Br}} \geq 0.7\right)$,

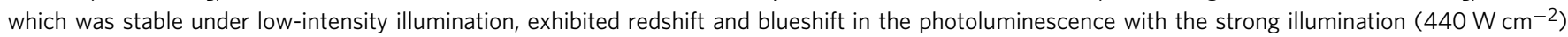



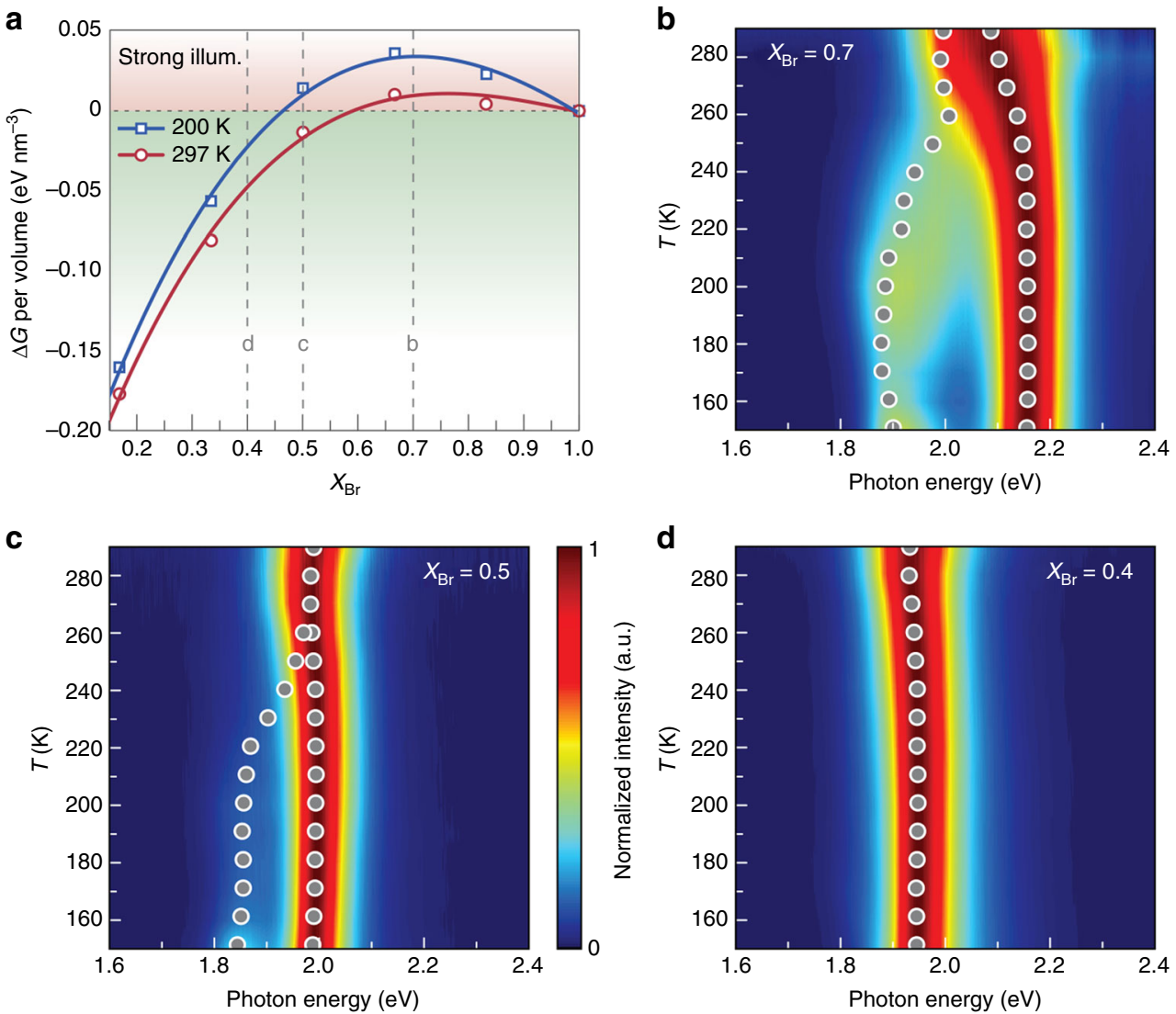

Fig. 4 Photo-stability of the perovskites exhibited strong temperature dependence. a The calculated $\Delta G_{\text {light }}$ indicates that, under strong illumination, the perovskite in the composites would be less photo-stable as the temperature reduces from $297 \mathrm{~K}$ to $200 \mathrm{~K}$. The vertical dash lines correspond to the compositions of samples examined experimentally in b-d. b-d Photoluminescence spectra of $\mathrm{CsPb}_{\mathrm{S}}\left(\mathrm{Br}_{\mathrm{x}} \mathrm{I}_{1-\mathrm{x}}\right)_{3} / \mathrm{Cs}_{4} \mathrm{~Pb}\left(\mathrm{Br}_{\mathrm{x}} \mathrm{I}_{1-\mathrm{x}}\right)_{6} \mathrm{composites}$ with $X_{\mathrm{Br}}=0.7(\mathbf{b})_{\text {, }}$

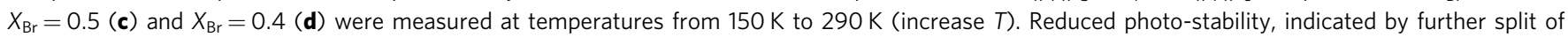
photoluminescence peaks, was observed at lower temperature in $\mathbf{b}$ and $\mathbf{c}$. The sample with higher iodine concentration in $\mathbf{d}\left(X_{\mathrm{Br}}=0.4\right)$ remained consistently stable across the entire temperature range, consistent with other measurements where I-rich perovskites appeared generally more photostable. The grey circles in $\mathbf{b}$-d show the peak positions determined by fitting the measured photoluminescence spectra. $\mathbf{b}$-d share the same color scale as shown in c

an explicit variable $T$ (Equation (1) \& (2)), should also be a function of the temperature. In general, $\Delta G_{\text {light }}$ increases when the temperature is reduced (Fig. 4a). Accordingly, an unstable mixed-halide perovskite could experience further phase separation as the temperature is lowered (Fig. 4a, dashed line b). Alternatively, samples that are photo-stable at room temperature could become unstable (Fig. 4a, dashed line c) or remain stable (Fig. $4 \mathrm{a}$, dashed line $\mathrm{d}$ ) at a lower temperature depending on the level of $\mathrm{Br}$ content $\left(X_{\mathrm{Br}}\right)$.

We were able to observe such temperature dependence experimentally by measuring the photoluminescence at different temperatures. Samples with three representative compositions $X_{\mathrm{Br}}=0.7,0.5,0.4$ (corresponding to the vertical dashed line $\mathrm{b} / \mathrm{c} / \mathrm{d}$ in Fig. 4a) were characterized. Given the high stability of the materials under low-intensity illumination, we had to use focused $405-\mathrm{nm}$ laser light $\left(440 \mathrm{~W} \mathrm{~cm}^{-2}\right)$ in order to reveal the evolution of photoluminescence with the temperature. Upon changing the temperature, no phase separation or abrupt changes have been observed in the initial spectra of photoluminescence, as shown by an example in Supplementary Figure 6. The spectra corresponding to final states were obtained after extended hours of illumination when no further evolution could be observed. For the most Br-rich perovskites (Fig. $4 \mathrm{~b}, X_{\mathrm{Br}}=0.7$ ), the photoluminescence peak split at room temperature after continuous optical illumination. The spectral separation between the photoluminescence peaks was found to increase continuously as the temperature was reduced from room temperature to $200 \mathrm{~K}$. The larger spectral split indicated higher iodine and bromine concentrations in the segregated domains, respectively. The reduced photo-stability could also be observed in the perovskite with nearly $1: 1 \mathrm{Br} / \mathrm{I}$ ratio (Fig. $4 \mathrm{c}, X_{\mathrm{Br}}=0.5$ ). While no spectral shift was observed at room temperature, a new peak corresponding to I-rich domains emerged as the temperature was reduced. Among the three samples, only the one with the highest iodine content remained homogeneous regardless of the temperature (Fig. $4 \mathrm{~d}, X_{\mathrm{Br}}=0.4$ ). The agreement between the theoretically predicted and the experimentally measured temperature dependence further validated the model of phase separation based on nucleation. Such phenomena could be attributed to the effect primarily driven by the changes of the entropy, which favor more uniformly distributed bromine and iodine anions (i.e. a homogenous phase) at elevated temperatures, as often observed in alloy systems $^{32}$.

\section{Discussion}

The stabilization of mixed-halide phases did not come at the cost of compromised optical properties. The photoluminescence quantum yields (PLQYs) of the composite thin films remained comparable with the previously reported bright emitters based on 
mixed-halide perovskites, with the highest reaching 36.8\% (Supplementary Figure $7 \mathrm{a}, X_{\mathrm{Br}}=0.5,620-\mathrm{nm}(2.0-\mathrm{eV})$ emission). The average photoluminescence lifetime was measured as 20 70 nanoseconds (ns), which appeared fairly long among the Csbased perovskite thin films reported (Supplementary Figure $7 \mathrm{~b}$ ). The addition of non-conductive $\mathrm{Cs}_{4} \mathrm{PbX}_{6}$ may alter the electrical properties from that of the thin films containing pure mixedhalide perovskites. Future studies of electrical properties will be necessary to optimize the optoelectronic functionalities of the inorganic composite perovskites by, for instance, finely tuning the molar ratio between the two component compounds in the composites.

\section{Methods}

Materials synthesis. An Edwards E306A coating system was used for dual-source evaporation. $\mathrm{Cs}$ and $\mathrm{Pb}$ salts $\left(\mathrm{CsBr}, \mathrm{CsI}, \mathrm{PbBr}_{2}, \mathrm{PbI}_{2}\right)$ were premixed with desirable ratios and used as precursor materials. Glass or thermal oxide substrates were cleaned sequentially by sonication in $1 \%$ Alconox precision cleaner solution, acetone, and isopropyl alcohol for $20 \mathrm{~min}$ followed by UV ozone treatment for 10 min prior to use. With a base pressure of $6 \times 10^{-6} \mathrm{mbar}$, the two precursor mixtures containing $\mathrm{Cs}$ and $\mathrm{Pb}$ salts were evaporated alternatingly from separate quartz crucibles. The evaporation rates were controlled $0.1-0.5 \mathrm{~A} \mathrm{~s}^{-1}$ for the $\mathrm{Pb}$ salts and 0.1-0.5 $\AA^{-1}$ for Cs salts. The above steps were repeated until the desired composition and film thickness were obtained (Supplementary Table 1). The evaporated samples were annealed at $150{ }^{\circ} \mathrm{C}$ on a hot plate in a nitrogen-filled glove box for $30 \mathrm{~min}$. Cesium bromide (99.9\%), cesium iodide (99.9\%), lead bromide (98\%) and lead iodide ( $98 \%)$ were acquired from Sigma-Aldrich.

Characterization. A PANalytical X'PERT Pro powder X-ray diffractometer with a $\mathrm{Cu} \mathrm{Ka}$ source was used to examine the crystal phases of the thin films after thermal annealing. The diffraction patterns were recorded from $2 \theta=10^{\circ}$ to $45^{\circ}$ with a step size of $0.0167^{\circ}$ at $0.8^{\circ} \mathrm{min}^{-1}$. TEM images were acquired using a JEM-ARM200cF operated at $200 \mathrm{kV}$. Samples for the TEM measurements were prepared by evaporating the thin films directly onto $\mathrm{Cu} /$ lacey-carbon TEM grids. Optical absorption spectra were measured using an Agilent Cary 5000 UV-Vis-NIR spectrometer. To avoid the exposure to the moisture in air, the samples were placed in a home-made airtight container with two sapphire windows. Photoluminescence spectra were measured using a HORIBA iHR320 spectrometer, equipped with a HORIBA Synapse back-illuminated CCD. The samples were placed in a vacuum chamber with cryogenic temperature control (Janis ST-500). A continuous-wave diode laser (405-nm) was used as the photoexcitation source. By focusing the laser beam to a 3- $\mu \mathrm{m}$ spot, the optical power density (i.e. intensity) could reach as high as $442.0 \mathrm{~W} \mathrm{~cm}^{-2}$.

DFT calculation. The Perdew-Burke-Ernzerhof $(\mathrm{PBE})^{36}$ exchange-correlation functional under the Generalized Gradient Approximation (GGA) and the projector augmented-wave (PAW) ${ }^{37}$ formalism were used for the total energy calculation. A plane-wave cutoff energy of $500 \mathrm{eV}$ and a $6 \times 6 \times 6 \mathrm{k}$-point mesh was used for all the configurations. The lattice volume and shape, and the atomic positions of each configuration were fully optimized using a quasi-Newton (variable metric) algorithm ${ }^{38}$ to minimize atomic forces below $1.0 \mathrm{meV}$ per $\AA$. The relaxed mixedhalide perovskite configurations at $X_{\mathrm{Br}}=0.5$ were shown in Supplementary Figure $5 \mathrm{a}$, where the degeneracy was five considering symmetry-reduced inequivalent configurations.

To determine the cohesive energy between pure $\mathrm{CsPbBr}_{3}$ and $\mathrm{CsPbI}_{3} W_{1}$ (equivalent to interfacial tension), we constructed a supercell $\left(2 \times 2 \times 2\right.$ of $\mathrm{CsPBBr}_{3}$ interfacing with $2 \times 2 \times 2$ of $\mathrm{CsPb}_{3}$ ) with an interface between the two pure materials, as shown in Supplementary Figure 5b. It is important to note that the cohesive energy contains contributions from: (i) the chemical interfacial energy due to chemical potential difference at the interface, and (ii) the strain energy at the interfaces due to lattice mismatch between the two crystals when they are constrained to the same lateral dimension in the supercell. The same DFT method and parameters mentioned above were used to relax this supercell, as well as to obtain the optimized configuration and the associated total energy at the ground state.

The similar DFT method was used to determine the cohesive energy $W_{2}$ between the initial $\mathrm{CsPb}\left(\mathrm{Br}_{\mathrm{x}} \mathrm{I}_{1-\mathrm{x}}\right)_{3}$ (nanocrystals 113) and $\mathrm{Cs}_{4} \mathrm{~Pb}\left(\mathrm{Br}_{\mathrm{x}} \mathrm{I}_{1-\mathrm{x}}\right)_{6}$ (matrix 416) phases, as well as the cohesive energy $W_{2}{ }^{\prime}$ between $\mathrm{Br}$-rich $\mathrm{CsPbBr}_{3}$ and $\mathrm{Cs}_{4} \mathrm{~Pb}\left(\mathrm{Br}_{\mathrm{x}} \mathrm{I}_{1-\mathrm{x}}\right)_{6}$ after phase separation. As shown in Supplementary Figure $5 \mathrm{c}$, the interface between the cubic $\mathrm{CsPbBr}_{3}$ phase (the (111) surface) and the hexagonal $\mathrm{Cs}_{4} \mathrm{PbI}_{6}$ phase (the (100) surface) is illustrated as an example. The $\mathrm{CsPbBr}_{3} /$ $\mathrm{Cs}_{4} \mathrm{PbBr}_{6}, \mathrm{CsPbBr}_{3} / \mathrm{Cs}_{4} \mathrm{PbI}_{6}, \mathrm{Cs} \mathrm{PbI}_{3} / \mathrm{Cs}_{4} \mathrm{PbI}_{6}$ and $\mathrm{CsPbI}_{3} / \mathrm{Cs}_{4} \mathrm{PbBr}_{6}$ interfaces were all relaxed and equilibrated using DFT calculations based on the reported crystallographic surfaces in an earlier work ${ }^{31}$.

\section{Data availability}

The authors declare that all the data supporting the findings of this study are available within the paper (and its supplementary information files). All the other relevant data are available from the corresponding author upon request.

Received: 9 August 2018 Accepted: 14 January 2019

Published online: 11 February 2019

\section{References}

1. Xing, G. et al. Low-temperature solution-processed wavelength-tunable perovskites for lasing. Nat. Mater. 13, 476-480 (2014).

2. Burschka, J. et al. Sequential deposition as a route to high-performance perovskite-sensitized solar cells. Nature 499, 316-319 (2013).

3. Fang, Y., Dong, Q., Shao, Y., Yuan, Y. \& Huang, J. Highly narrowband perovskite single-crystal photodetectors enabled by surface-charge recombination. Nat. Photonics 9, 679-686 (2015).

4. Stranks, S. D. et al. Electron-hole diffusion lengths exceeding 1 micrometer in an organometal trihalide perovskite absorber. Science 342, 341-344 (2013).

5. Becker, M. A. et al. Bright triplet excitons in cesium lead halide perovskites. Nature 553, 189-193 (2018).

6. Shi, D. et al. Low trap-state density and long carrier diffusion in organolead trihalide perovskite single crystals. Science 347, 519-522 (2015).

7. Tsai, H. et al. Light-induced lattice expansion leads to high-efficiency perovskite solar cells. Science 360, 67-70 (2018).

8. Zhu, H. et al. Lead halide perovskite nanowire lasers with low lasing thresholds and high quality factors. Nat. Mater. 14, 636-642 (2015).

9. Sahli, F. et al. Fully textured monolithic perovskite/silicon tandem solar cells with 25.2\% power conversion efficiency. Nat. Mater. 9, 1552 (2018).

10. Yang, W. S. et al. Iodide management in formamidinium-lead-halidebased perovskite layers for efficient solar cells. Science 356, 1376-1379 (2017).

11. Kojima, A., Teshima, K., Shirai, Y. \& Miyasaka, T. Organometal halide perovskites as visible-light sensitizers for photovoltaic cells. J. Am. Chem. Soc. 131, 6050-6051 (2009).

12. Kim, H.-S. et al. Lead iodide perovskite sensitized all-solid-state submicron thin film mesoscopic solar cell with efficiency exceeding 9\%. Sci. Rep. 2, 583-587 (2012).

13. Zhou, H. et al. Interface engineering of highly efficient perovskite solar cells. Science 345, 542-546 (2014)

14. Tan, Z.-K. et al. Bright light-emitting diodes based on organometal halide perovskite. Nat. Nanotech 9, 687-692 (2014).

15. Cho, H. et al. Overcoming the electroluminescence efficiency limitations of perovskite light-emitting diodes. Science 350, 1222-1225 (2015).

16. Ling, Y. et al. Enhanced optical and electrical properties of polymer-assisted all-inorganic perovskites for light-emitting diodes. Adv. Mater. 28, 8983-8989 (2016).

17. Song, J. et al. Quantum dot light-emitting diodes based on inorganic perovskite cesium lead halides (CsPbX3). Adv. Mater. 27, 7162-7167 (2015).

18. Xiao, Z. et al. Mixed-halide perovskites with stabilized bandgaps. Nano. Lett. 17, 6863-6869 (2017).

19. Protesescu, L. et al. Nanocrystals of cesium lead halide perovskites (CsPbX3, X $=\mathrm{Cl}, \mathrm{Br}$, and I): novel optoelectronic materials showing bright emission with wide color gamut. Nano. Lett. 15, 3692-3696 (2015).

20. Zhang, D. et al. Synthesis of composition tunable and highly luminescent cesium lead halide nanowires through anion-exchange reactions. J. Am. Chem. Soc. 138, 7236-7239 (2016).

21. Anaya, M., Lozano, G., Calvo, M. E. \& Míguez, H. ABX3 Perovskites for tandem solar cells. Joule 1, 769-793 (2017).

22. Hoke, E. T. et al. Reversible photo-induced trap formation in mixed-halide hybrid perovskites for photovoltaics. Chem. Sci. 6, 613-617 (2014).

23. Beal, R. E. et al. Cesium lead halide perovskites with improved stability for tandem solar cells. J. Phys. Chem. Lett. 7, 746-751 (2016).

24. Bischak, C. G. et al. Origin of reversible photoinduced phase separation in hybrid perovskites. Nano. Lett. 17, 1028-1033 (2017).

25. Yoon, S. J. et al. Tracking iodide and bromide ion segregation in mixed halide lead perovskites during photoirradiation. ACS Energy Lett. 1, 290-296 (2016).

26. Wang, Y. et al. Epitaxial halide perovskite lateral double heterostructure. ACS Nano. 11, 3355-3364 (2017).

27. Slotcavage, D. J., Karunadasa, H. I. \& McGehee, M. D. Light-induced phase segregation in halide-perovskite absorbers. ACS Energy Lett. 1, 1199-1205 (2016). 
28. Brennan, M. C., Draguta, S., Kamat, P. V. \& Kuno, M. Light-induced anion phase segregation in mixed halide perovskites. ACS Energy Lett. 3, 204-213 (2017).

29. Draguta, S. et al. Rationalizing the light-induced phase separation of mixed halide organic-inorganic perovskites. Nat. Commun. 8, 200 (2017)

30. Lin, Q., Wang, Z., Snaith, H. J., Johnston, M. B. \& Herz, L. M. Hybrid perovskites: prospects for concentrator solar cells. Adv. Sci. 5, 1700792-1700798 (2018).

31. Quan, L. N. et al. Highly emissive green perovskite nanocrystals in a solid state crystalline matrix. Adv. Mater. 29, 1605945-1605946 (2017).

32. Porter, D. A. \& Easterling, K. E. Phase Transformations in Metals and Alloys. (Springer US, Boston, 1992).

33. Miyata, K. et al. Large polarons in lead halide perovskites. Sci. Adv. 3, e1701217 (2017)

34. Batignani, G. et al. Probing femtosecond lattice displacement upon photocarrier generation in lead halide perovskite. Nat. Commun. 9, 1971 (2018).

35. Bretschneider, S. A. et al. Quantifying polaron formation and charge carrier cooling in lead-iodide perovskites. Adv. Mater. 338, 1707312-1707318 (2018).

36. Perdew, J. P., Burke, K. \& Ernzerhof, M. Generalized gradient approximation made simple. Phys. Rev. Lett. 77, 3865-3868 (1996).

37. Blochl, P. E. Projector augmented-wave method. Phys. Rev. B 50, 17953-17979 (1994).

38. Pulay, P. Convergence acceleration of iterative sequences-the case of scf iteration. Chem. Phys. Lett. 73, 393-398 (1980).

\section{Acknowledgements}

The authors thank Dr. Eric Lochner, Mr. Dong Li, Dr. Biwu Ma, Dr. Peng Xiong, Mr. Kurt Koetz, and the staff of the Physics Machine Shop for their help with the experiments. This work used equipment in the Physics Department CMMP User Facility and the Chemistry Department MAC Laboratory. The TEM measurements were performed in the Electron Microscopy Facility co-funded by the National Science Foundation (DMR-1644779) and the State of Florida. This work was financially supported by Florida State University through the Energy and Materials Initiative and by Office of Naval Research through the Young Investigator Program (N00014-18-1-2408). S.L. and K.B.D. acknowledge financial support from NSF (CBET-1708968) and the Startup Funds from Florida State University. All data is available in the main text or the supplementary materials.

\section{Author contributions}

X.W., Y.L. and H.G. conceived the idea and designed experiments; X.W., Y.L., F.P-O., J. K. prepared materials; Y.X. performed TEM measurements and analysis; X.W., X.L., Z.C., Y.Z. and D.B. performed characterizations and analyses; X.W., H.G., S.L., and J.S. proposed the nucleation model; K.B.D. and S.L carried out DFT calculations; X.W., S.L., J.S. and H.G. interpreted the data; X.W., S.L., Y.X., J.S., K.H., and H.G. prepared the manuscript.

\section{Additional information}

Supplementary Information accompanies this paper at https://doi.org/10.1038/s41467019-08610-6.

Competing interests: The authors declare no competing interests.

Reprints and permission information is available online at http://npg.nature.com/ reprintsandpermissions/

Journal peer review information: Nature Communications thanks the anonymous reviewers for their contribution to the peer review of this work.

Publisher's note: Springer Nature remains neutral with regard to jurisdictional claims in published maps and institutional affiliations.

Open Access This article is licensed under a Creative Commons Attribution 4.0 International License, which permits use, sharing, adaptation, distribution and reproduction in any medium or format, as long as you give appropriate credit to the original author(s) and the source, provide a link to the Creative Commons license, and indicate if changes were made. The images or other third party material in this article are included in the article's Creative Commons license, unless indicated otherwise in a credit line to the material. If material is not included in the article's Creative Commons license and your intended use is not permitted by statutory regulation or exceeds the permitted use, you will need to obtain permission directly from the copyright holder. To view a copy of this license, visit http://creativecommons.org/ licenses/by/4.0/.

(c) The Author(s) 2019 